\title{
Synthesis and Biological Activity of Novel Thieno $[2,3-d][1,2,4]$ triazolo $[4,3-a]$ pyrimidine and Pyrazolo [3,4-c] pyrazole Derivatives
}

\author{
Asma M. Mahran*, Khadiga M. Abu-Zied and Wafaa A. Gad
}

Photochemistry Department, National Research Center, Giza, Egypt.

\begin{abstract}
D EACTION of hydrazonoyl halides (2a-e) with either 1,2,3,5,6,7hexahydro- $4 H$-cyclopenta[4,5]thieno[2,3- $d$ ] pyrimidin-2, 4-dithione (1) or 2 (4- Dihydro -5 - oxo -3 - methyl - $1 H$ - pyrazolo $-1-y l$ ) $-5,6,7,8$-tetrahydrothieno[2,3-d]pyrimidin- $4(3 H, 4 H)$-one $(11)$ in the presence of sodium ethoxide in refluxing ethanol / DMF mixture afforded the cyclopenta [4,5] thieno[2,3- $d]$ triazolo [4,3- $a]$ pyrimidines4-thiones (7a-d) and the phenylpyrazolo[3,4-c]pyrazolcyclopenta $[4,5]$ thieno $[2,3-d]$ pyrimidin-4-ones (14a-e), respectively. The mechanism of the studied reactions has been discussed and the biological activity of the products $7 \mathrm{a}-\mathrm{d}$ has been evaluated.
\end{abstract}

Keywords: Hydrazonoyl halides, [1,2,4] Triazolopyrimidine, Rearrangement , Pyrazole and Biological activities.

The biological ${ }^{(1-3)}$, bactericidal $^{(4)}$ and medicinal ${ }^{(5,6)}$ activities of theino[2,3- $\left.d\right]$ pyrimidine derivatives have recently attracted considerable attention ${ }^{(7,8)}$. In view of the above findings and in continuation to our interest on the use of hydrazonoyl halides for the synthesis of heterocyclic compounds incorporating different functionalities of biological importance ${ }^{(9,10)}$ we reported here a facile and short synthesis of the title compounds starting from either 2,4-thioxo1,2,3,4,5,6,7-hexahydro-4H-cyclopenta[4,5] thieno[2,3- $d$ ] pyrimidin-2,4-dithione (1) or 2(4-dihydro-5-oxo-3-methyl-1 $H$-pyrazolo-1-yl)-5,6,7,8-tetrahydrothieno [2,3- $d]$ pyrimidin- $4(3 H, 1 H)$-one (11) and hydrazonoyl halides (2) .

\section{Results and Discussion}

Reaction of 3,5,6,7-hexahydrocyclopenta[4,5]thieno[2,3- $d$ ] pyrimidin-2,4dithione (1) which was prepared from 3-(2-amino-thiophene)-carbonitrile derivatives with carbon disulfide in dry pyridine as previously reported ${ }^{(11)}$ with hydrazonoyl halides (2a-d) ${ }^{(12-15)}$ in sodium ethoxide in refluxing ethanol for 7-10 $\mathrm{hr}$ afforded a single product as evidenced by TLC. The structures of the isolated products were established by analytical and spectroscopic data (MS, IR and

*E-mail: dr_asma_chem@yahoo.com 
${ }^{1} \mathrm{H}$ NMR) and identified as cyclopenta[4,5]thieno[2,3- $\left.d\right]$ triazolo[4,3- $a$ ]pyrimidin5-thiones (7a-d).Their mass spectra revealed in each case the respective molecular ion peak and the $\left(\mathrm{M}^{+}+1\right)$ peak for example 7 a gave molecular ion peak at $\mathrm{m} / \mathrm{z} 366$ corresponding to $\mathrm{C}_{18} \mathrm{H}_{14} \mathrm{~N}_{4} \mathrm{OS}_{2}$. The IR spectra of each of the studied compounds 7a-d revealed the absence of the $\mathrm{NH}$ absorption band around $3422 \mathrm{~cm}^{-1}$. The IR spectrum of 7 a showed three absorption bands at $1652(\mathrm{C}=\mathrm{O})$, $1245(\mathrm{C}=\mathrm{S})$ and $3170(\mathrm{CH}-\mathrm{Ar}) \mathrm{cm}^{-1}$. Furthermore the ${ }^{1} \mathrm{HNMR}$ spectra of $7 \mathrm{a}-\mathrm{d}$ are characterized by the absence of signal near $\delta: 10.8-12.00 \mathrm{ppm}$ characteristic of $\mathrm{NH}$ and the appearance of signal near 7.00-8.00 ppm (Ar-H) (see Experimental).

To account for the direct formation of the latter products $7 \mathrm{a}-\mathrm{d}$, the mechanism outlined in Scheme 1 is proposed. It suggested that the studied reactions involved an initial formation of thiohydrazonate esters (3), which undergo $\mathrm{S}$ to $\mathrm{N}$ migration to give the thiohydrazides (6)via the Spiro-adduct $(5)^{(16)}$. In all cases examined attempts to isolate the intermediate 3-6 failed however, this finding indicates that such intermediates are consumed as soon as they are formed under the reaction conditions employed as they readily undergo in situ cyclization followed by elimination of hydrogen sulfide to give final products (7a-d).

The assignment of the structure 7 were further substantiated by an alternate synthesis of 7 based on synthesis of the thiohydrazonate ester (3) via application of Japp-Klingemann reaction ${ }^{(16,17)}$ to the active methine compounds of type 9. The latter compound is prepared in this work by reaction of 1,2,3,5,6,7hexahydrocyclopenta [4,5] thieno [2,3- $d$ ] pyrimidin-2,4-dithione (1) with $\alpha$ chloroacetoacetanilide (compound 8) in ethanol / DMF in the presence of triethylamine. The structure of $9 \mathrm{c}$ is evidenced by its microanalyses and spectra (MS, IR and ${ }^{1} \mathrm{H}$ NMR) (see Experimental). For example, its ${ }^{1} \mathrm{H}$ NMR spectra showed signal for the methine hydrogen at $5.10 \mathrm{ppm}$ assignable to - $\mathrm{SCH}$ proton which is lost on shaking the solution with deuterium oxide showing its ready exchangeability through enolization, also, a signal at $2.37 \mathrm{ppm}$ assignable to $\mathrm{COCH}_{3}$. Next, coupling of $9 \mathrm{c}$ with diazotized aniline was investigated in ethanol in the presence of sodium acetate at low temperature $\left(0-5 C^{\circ}\right)$. On the basis of previous literature on coupling of active chloromethylene compounds with diazonium salts ${ }^{(17)}$, we anticipated that reactions of $9 \mathrm{c}$ with diazotized aniline would give the thiohydrazonate ester (3c) directly via cleavage of the acetyl group from 10c (Scheme 1). However, in our hands such reactions yielded product proved to be identical in all respects (m.p., mixed m.p. and IR spectra) with 7c obtained above.

Egypt. J. Chem. 55, No. 2 (2012) 


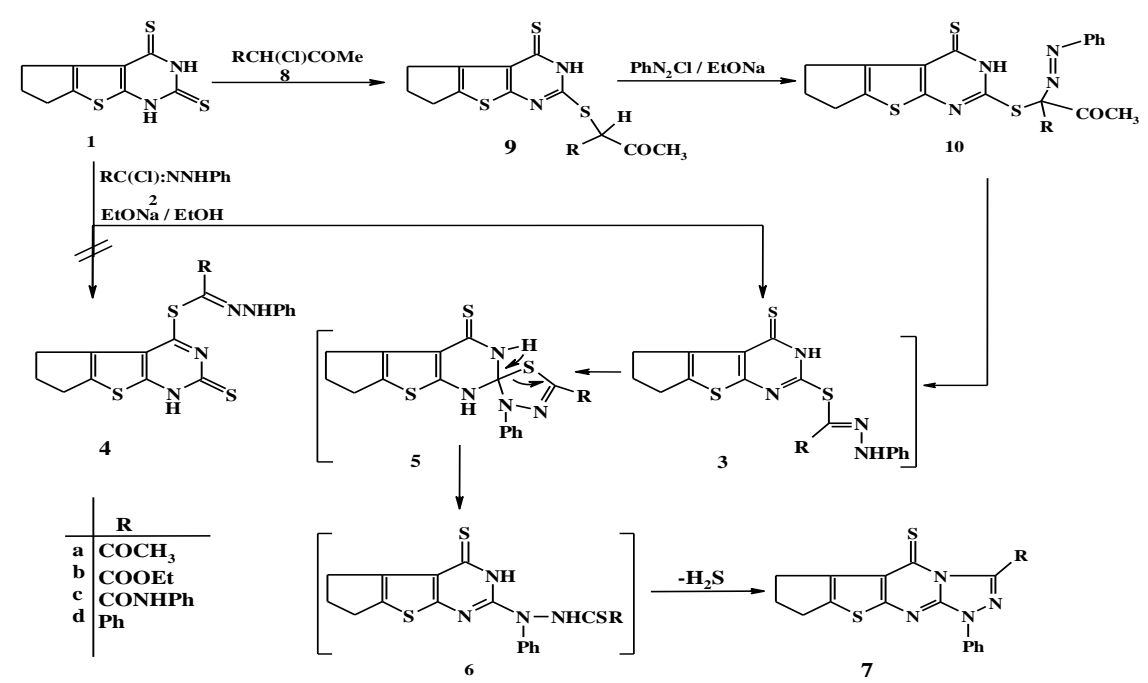

Scheme 1

Also, hydrazonoyl halides (2a-e) reacted with 2(4-Dihydro-5-oxo-3-methyl$1 H$-pyrazolo-1-yl)-5,6,7,8-tetrahydrothieno[2,3-d]pyrimidin-4 $(3 H, 1 H)$-one $(11)^{(18)}$ in sodium ethoxide in refluxing ethanol/DMF mixture, after work up of the reaction mixtures only a single product was isolated in each case 14 .

The structure of 14 was assigned to the reaction product based on spectral and elemental analysis. For example the IR spectra of 14a displayed absorption bands at 1700, $1670(2 \mathrm{CO})$ and $3422 \mathrm{~cm}^{-1}(\mathrm{NH})$ (see Experimental) which provided no additional evidence for cyclic products at $\mathrm{NH}$ group. Also, its ${ }^{1} \mathrm{H}$ NMR spectra showed disappearance of signals at $3.27 \mathrm{ppm}$ assignable to $\left(\mathrm{CH}_{2}\right)$ group and showed a signal at $2.25 \mathrm{ppm}\left(\mathrm{COCH}_{3}\right)$ and 7.28-8.14 ppm for aromatic protons. Moreover, its mass spectrum gave $\mathrm{m} / \mathrm{z}=430$ corresponding to the molecular weight of molecular formula $\mathrm{C}_{22} \mathrm{H}_{18} \mathrm{~N}_{6} \mathrm{O}_{2} \mathrm{~S}$ of the assigned structure.

To account for the formation of 14 from 11 with 2 , the two step reaction sequence outlined in Scheme 2 is suggested. The first step involves nucleophilic attack of 2 on the active methylene of 11 to give the substitution products $12 \mathrm{a}-\mathrm{d}$ or 13a-d, respectively. The second step in the suggested mechanism (Scheme 2) involves cyclization of the latter intermediates through dehydration of $13 \mathrm{a}$-e to give 14a-e as end product (Scheme 2).

Attempts to isolate one of each of the open chain intermediate 12 or 13 under mild conditions were successes. For example we succeeded in isolating one of such intermediate. Reaction of 11 with $2 \mathrm{a}$ in ethanol in the presence of triethylamine at room temperature afforded $12 \mathrm{a}$. The structure of the latter was established on the basis of microanalyses and spectral data (IR, MS and

Egypt. J. Chem. 55, No. 2 (2012) 
${ }^{1} \mathrm{H}$ NMR). When the latter was refluxed in ethanolic sodium ethoxide solution, it yielded 14; this finding indicaties that 12a-d and 13a-d are intermediates in the studied reactions and are consumed as they are formed .
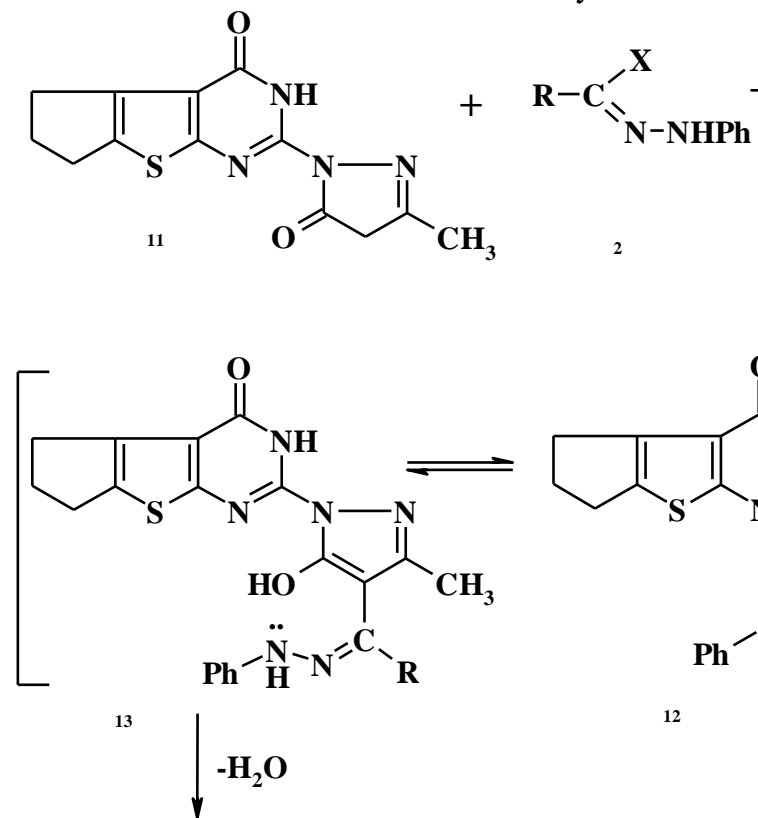<smiles>[R]/C(=N/N=Nc1ccccc1)C(=O)n1nc(C)c(=O)n1-c1nc2sc3c(c2c(=O)[nH]1)CCC3</smiles><smiles>[R]c1nn(-c2ccccc2)c2c1c(C)nn2-c1nc2sc3c(c2c(=O)[nH]1)CCC3</smiles>

\begin{tabular}{l|l} 
& $\mathrm{R} / \mathrm{X}$ \\
\hline $\mathrm{A}$ & $\mathrm{COCH}_{3} / \mathrm{Cl}$ \\
$\mathrm{B}$ & $\mathrm{COOCH}_{2} \mathrm{CH}_{3} / \mathrm{Cl}$ \\
$\mathrm{C}$ & $\mathrm{CONHPh} / \mathrm{Cl}$ \\
$\mathrm{D}$ & $\mathrm{Ph} / \mathrm{Cl}$ \\
$\mathrm{E}$ & $\mathrm{COPh} / \mathrm{Br}$
\end{tabular}

Biological activity

Scheme 2

Antimicrobial activities of the tested compounds 7a-d were determined using a modified Kirby-Bauer disk diffusion method ${ }^{(19)}$. Plates inoculated with filamentous fungi as Aspergillus flavus at $25{ }^{\circ} \mathrm{C}$ for $48 \mathrm{hr}$; Gram (+) bacteria as Staphylococcus aureus and Bacillus subtilis; Gram (-) bacteria as Escherichia coli and Pseudomonas aeuroginosa were incubated at $35-37^{\circ} C$ four $24-48 \mathrm{hr}$ and yeast as Candida albicans incubated at $30^{\circ} \mathrm{C}$ for $24-48 \mathrm{hr}$ and then the diameters of the inhibition zones were measured in millimeters ${ }^{(19)}$. Standard discs of Tetracycline (antibacterial agent), Amphotericin B (antifungal agent) served as positive controls for antimicrobial activity. But filter discs impregnated with 10uI of solvent (distilled water, chloroform DMSO) were used as negative

Egypt. J. Chem. 55, No. 2 (2012) 
control. The results showed that all the tested compounds are highly active as antifungal agents compared to the reference (Amphotericin B) to the Gram (+) bacteria (Staphylococcus aureus) ${ }^{(20)}$. They have antibacterial activity less than tetracycline .The results are summarized in Table 1.

\begin{tabular}{|c|c|c|c|c|c|}
\hline & \multirow[b]{2}{*}{ Sample } & \multicolumn{4}{|c|}{ Inhibition zone diameter ( $\mathrm{mm} / \mathrm{mg}$ sample) } \\
\hline & & $\begin{array}{c}\text { Escherichia } \\
\text { coli } \\
\left(G^{-}\right) \\
\end{array}$ & $\begin{array}{c}\text { Staphylococcus } \\
\text { aureus } \\
\left(\mathbf{G}^{+}\right) \\
\end{array}$ & $\begin{array}{c}\text { Aspergillus } \\
\text { flavus } \\
\text { (Fungus) } \\
\end{array}$ & $\begin{array}{c}\text { Candida } \\
\text { albicans } \\
\text { (Fungus) } \\
\end{array}$ \\
\hline \multicolumn{2}{|c|}{ Control: DMSO } & 0.0 & 0.0 & 0.0 & 0.0 \\
\hline \multirow{2}{*}{ 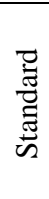 } & $\begin{array}{l}\text { Tetracycline } \\
\text { Antibacterial } \\
\text { agent }\end{array}$ & 30 & 29 & -- & -- \\
\hline & $\begin{array}{l}\text { Amphotericin B } \\
\text { Antifungal agent }\end{array}$ & -- & -- & 19 & 21 \\
\hline & $7 \mathrm{a}$ & 0.0 & 14 & 0.0 & 0.0 \\
\hline & $7 \mathrm{~b}$ & 12 & 13 & 0.0 & 0.0 \\
\hline & $7 \mathrm{c}$ & 13 & 14 & 0.0 & 0.0 \\
\hline & $7 \mathrm{~d}$ & 0.0 & 12 & 0.0 & 0.0 \\
\hline
\end{tabular}

G: Gram reaction.

The samples have antibacterial activity but don't have any antifungal activity on tested microorganisms.

\section{Experimental}

Melting points were determined on a Gallenkamp apparatus and are uncorrected, IR spectra were recorded in potassium bromide using PU 9712 spectrophotometer, H NMR spectra were recorded in deuterated chloroform using a Varian Gemini 300 NMR spectrometer. Mass spectra were recorded on a 75 Kratos spectrometer. Elemental analyses were carried out at the Micro Analytical Laboratory of National Research Center, Giza, Egypt. Biological activity was carried out at the Microbiology Laboratory of Faculty of ScienceCairo University.

Synthesis of $7 a-d$ and $14 a-e$

General procedure

To a stirred solution of each of 1 or 11 (10 mmoles) in ethanol sodium ethoxide solution, prepared by dissolving sodium metal $(0.23 \mathrm{~g}, 10 \mathrm{mmoles})$ in absolute ethanol $(50 \mathrm{ml})$ and few drops of DMF, was added the appropriate hydrazonoyl halides $2 \mathrm{~A}-\mathrm{E}$ (1mmoles). The reaction mixture was heated under reflux for 6-8 hr (monitored with TLC). The reaction mixture was then cooled 
and the solid that precipitated was filtered off and crystallized from the proper solvent to give $7 \mathrm{a}-\mathrm{d} \& 14 \mathrm{a}-\mathrm{e}$, respectively. The physical constants of the compounds prepared are given below.

3-Acetyl-1-phenyl-cyclopenta [4,5] thieno [2,3-d] triazolo [4,3-a] pyrimidin5(1H,5H)-5-thione (7a): white crystals, Yield $(2.56 \mathrm{~g}, 70 \%)$, m.p.162-164C $(\mathrm{EtOH}) ; \mathrm{IR}(\mathrm{KBr}) \mathrm{v} \mathrm{cm}^{-1}$ : (3170,CH-Ar), 1652(CO),1245(C=S). MS m/z (\%): 366 $\left(\mathrm{M}^{+}, 91\right), 367\left(\mathrm{M}^{+}+1\right) .{ }^{1} \mathrm{H}$ NMR (DMSO-d $\left.{ }_{6}\right) \delta \mathrm{ppm}: 2.12\left(\mathrm{t}, 2 \mathrm{H}, \mathrm{CH}_{2}\right), 2.28(\mathrm{~s}$, $\left.3 \mathrm{H}, \mathrm{CH}_{3}\right), 2.50\left(\mathrm{~m}, 4 \mathrm{H}, 2 \mathrm{CH}_{2}\right), 2.72\left(\mathrm{t}, 2 \mathrm{H}, \mathrm{CH}_{2}\right), 7.23-7.95(\mathrm{~m}, 5 \mathrm{H}, \mathrm{Ar}-\mathrm{H})$; Anal. Calcd. For $\mathrm{C}_{18} \mathrm{H}_{14} \mathrm{~N}_{4} \mathrm{OS}_{2}$ (366.47): C, 59.00; H, 3.85; N, 15.29, 9.13; S, $17.50 \%$. Found: C, 59.00; H, 3.90; N, 15.13; S, 17.47\%.

3-Ethoxycarbonyl-1-phenyl-cyclopenta[4,5]thieno[2,3-d]triazolo[4,3-a] pyrimidin5(1H,5H)-5-thione $(7 \mathrm{~b})$ : Pale brown crystals, Yield $(2.65 \mathrm{~g}, 72 \%)$ m.p. $\left(169^{\circ}\right.$ $170) \mathrm{C}^{0}(\mathrm{EtOH}) . \mathrm{IR}(\mathrm{KBr}) \mathrm{v} \mathrm{cm}^{-1}: 3170(\mathrm{CH}-\mathrm{Ar}), 1735(\mathrm{CO}), 1245(\mathrm{C}=\mathrm{S}) . \mathrm{MS} \mathrm{m} / \mathrm{z}$ $(\%): 396\left(\mathrm{M}^{+}, 73\right), 397\left(\mathrm{M}^{+}+1\right) .{ }^{1} \mathrm{H} \mathrm{NMR}\left(\mathrm{CDCl}_{3}\right) \delta \mathrm{ppm}: 1.23-1.28\left(\mathrm{t}, 3 \mathrm{H}, \mathrm{CH}_{3}\right)$, 2.20(t, 3H, $\left.\mathrm{CH}_{2}\right), 2.51\left(\mathrm{~m}, 4 \mathrm{H}, 2 \mathrm{CH}_{2}\right), 4.11-4.30\left(\mathrm{q}, 2 \mathrm{H}, \mathrm{CH}_{2} \mathrm{CH}_{3}\right), 7.12-7.49(\mathrm{~m}$, 5H, Ar-H). Anal. Calcd. For $\mathrm{C}_{19} \mathrm{H}_{16} \mathrm{~N}_{4} \mathrm{O}_{2} \mathrm{~S}_{2}$ (396.49): C, 57.56: H, 4.07; N, 14.13; S, $16.17 \%$. Found: C, 58.09; H, 4.02; N, 14.11; S, 16.09\%.

\section{Synthesis of $7 c$}

To a solution of 9 (10 m mole) in ethanol/DMF was added sodium acetate trihydrate $(3 \mathrm{~g})$ and the mixture was cooled in an ice bath to $0-5^{\circ} \mathrm{C}$ while being stirred. To the resulting cold solution was added portionwise a cold solution of benzenediazonium chloride, prepared as usual by diazotizing aniline (10mmole) in hydrochloric acid $(6 \mathrm{ml}, 6$ moles $)$ with sodium nitrite $(0.7 \mathrm{~g}, 10 \mathrm{~m}$ mole $)$ in water $(10 \mathrm{ml})$. After all diazonium salt added, the reaction mixture was stirred for $1 \mathrm{hr}$ while cooling in ice bath and left overnight in a refrigerator. The solid that precipitated was filter washed with water, air dried and finally crystallized by ethanol.

$N$-Phenyl-1-phenyl-cyclopenta [4,5] thieno [2,3-d]triazolo[4,3-a]pyrimidin-5 (1H,5H)-5-thione -3-carboxamide (7c): Pale brown, Yield (2.96g,67\%), m.p.136-138 ${ }^{\circ} \mathrm{C}(\mathrm{EtOH}) . \quad \mathrm{IR}(\mathrm{KBr}) v \mathrm{~cm}^{-1}$ : 3130 (CH-phenyl), $3222(\mathrm{NH})$, 1610(CO), 1360(C=S). MS m/z (\%): 443( $\left.\mathrm{M}^{+}+61\right)$ 444( $\left(\mathrm{M}^{+}+1\right) .{ }^{1} \mathrm{H} \mathrm{NMR}\left(\mathrm{CDCl}_{3}\right)$ $\delta$ ppm: 1.72(m, $\left.4 \mathrm{H}, 2 \mathrm{CH}_{2}\right), 2.82\left(\mathrm{t}, 2 \mathrm{H}, \mathrm{CH}_{2}\right), 3.01\left(\mathrm{t}, 2 \mathrm{H}, \mathrm{CH}_{2}\right), 7.12-8.10(\mathrm{~m}$, $10 \mathrm{H}, 2 \mathrm{Ar}-\mathrm{H}), 8.64(\mathrm{~s}, 1 \mathrm{H}, \mathrm{NH}) .{ }^{13} \mathrm{C} \mathrm{NMR}\left(\mathrm{CDCl}_{3}\right) \delta \mathrm{ppm}: \quad 28.2,29.9,32.3$, $115.1,120.7,121.3,125.2,127.8,129.1,129.3,135.2,136.1,136.6,137.8$, 139.6, 145.3, 151.6, 154.8, 177.8. Anal. Calcd. For $\mathrm{C}_{23} \mathrm{H}_{17} \mathrm{~N}_{5} \mathrm{OS}_{2}$ (443.55): C, 62. $28 ; \mathrm{H}, 3.86 ; \mathrm{N}, 15.79 ; \mathrm{S}, 14.46 \%$. Found: C, 61.97; H, 3.77; N, 15.69; S, $14.41 \%$.

1,3-Diphenyl-cyclopnenta[4,5]thieno[2,3-d]triazolo[4,3-a]pyrimidin -5(1H,5H)-5thione (7d): balk crystals, Yield $(2.80 \mathrm{~g}, 72 \%)$ m.p. $261^{\circ} \mathrm{C}(\mathrm{EtOH} / \mathrm{Dioxane})$. IR (KBr) v cm ${ }^{-1}: 3012$ (CH-Ar), 1245(C=S), . MS m/z (\%): 400( $\left.\mathrm{M}^{+}, 100\right) .{ }^{1} \mathrm{H}$ NMR $\left(\mathrm{DMSO}_{6}\right) \delta$ ppm: $1.51\left(\mathrm{~m}, 4 \mathrm{H}, 2 \mathrm{CH}_{2}\right), 2.1\left(\mathrm{t}, 2 \mathrm{H}, \mathrm{CH}_{2}\right), 2.62\left(\mathrm{t}, 2 \mathrm{H}, \mathrm{CH}_{2}\right), 7.10-$ 
7.39 (m, 10H, Ar-H). Anal. Calcd. For $\mathrm{C}_{22} \mathrm{H}_{16} \mathrm{~N}_{4} \mathrm{~S}_{2}$ (400.53): C, 65.97; H, 4.03; N, 13.99; S, 16.01\%. Found: C, 65.88; H, 4.00; N, 13.79; S, $16.00 \%$.

\section{Synthesis of 9}

To a mixture of equimolar quantities of $\alpha$-chloroacetoacetanilide (8) and 3,5,6,7-hexahydrocyclopenta [4,5] thieno [2,3- $d]$ pyrimidin - 2,4 - dithione 1 (10 mmoles) in absolute ethanol/DMF mixture $(40 \mathrm{ml})$ was added triethylamine $(1.4$ $\mathrm{ml}, 10$ mmoles).The mixture was stirred for two days at room temperature. During this period the reactants dissolved. The solvent was evaporated under reduced pressure. The oily residue left was triturated with methanol and left in a refrigerator overnight. The solid that was produced was collected by filtration and crystallized to give 9 in good yield.

N-Phenyl-3-thio-2-[1,2,3,5,6,7-hexahydrocyclopenta[4,5]thieno [2,3-d] pyrimidin4-thione] Butanamide (9)

Pale black, Yield (2.82 g, 68\%), m.p.180-182 ${ }^{\circ} \mathrm{C}$ (EtOH/Dioxane). IR $(\mathrm{KBr}) v \mathrm{~cm}^{-1}:$ 3422(NH), 3129(CH-Ar), 2920(CH), 1627, 1650, 1243(C=S). MS $\mathrm{m} / \mathrm{z}(\%): 415\left(\mathrm{M}^{+}, 70\right) .{ }^{1} \mathrm{H}$ NMR (DMSO-d $\left.\mathrm{d}_{6}\right) \delta \mathrm{ppm}: 1.35\left(\mathrm{~m}, 4 \mathrm{H}, 2 \mathrm{CH}_{2}\right), 1.84(\mathrm{~S}$, $3 \mathrm{H}, \mathrm{CH} 3), 2.80\left(\mathrm{t}, 2 \mathrm{H}, \mathrm{CH}_{2}\right), 3.25\left(\mathrm{t}, 2 \mathrm{H}, \mathrm{CH}_{2}\right), 5,01(\mathrm{~S}, 1 \mathrm{H}, \mathrm{CH}), 7.01-7.81(\mathrm{~m}, 5 \mathrm{H}$, Ar-H). Anal. Calcd. For $\mathrm{C}_{19} \mathrm{H}_{17} \mathrm{~N}_{3} \mathrm{O}_{2} \mathrm{~S}_{3}$ (415.56): C, 54.92; H, 4.12; N, 10.11; S, 23.15\%. Found: C, 54.89; H, 4.08; N, 10.04; S, $23.04 \%$.

\section{Synthesis of the intermediate $12 a$}

To a mixture of 11 and $2 \mathrm{a}(0.01 \mathrm{~mol}$ each $)$ in ethanol $(50 \mathrm{ml})$ and few drops from DMF, was added triethylamine $(1.4 \mathrm{ml}, 0.01 \mathrm{~mol})$ and the reaction mixture was stirred for $3 \mathrm{hr}$ at room temperature. The solid that precipitated was filtered off and crystallized from (EtOH) to give $12 \mathrm{a} ;{ }^{1} \mathrm{HNMR}\left(\mathrm{CDCl}_{3}\right) \delta \mathrm{ppm}$ : pale brown, $(2.69 \mathrm{~g}, 60 \%)$, m.p. $148{ }^{\circ} \mathrm{C}$. IR (KBr) v cm $\mathrm{cm}^{-1}: 3432,3385,1668$. MS m/z (\%): 448( $\left.\mathrm{M}^{+}, 22\right) .{ }^{1} \mathrm{HNMR}\left(\mathrm{CDCl}_{3}\right) \delta \mathrm{ppm}: 2.11\left(\mathrm{~s}, 3 \mathrm{H}, \mathrm{CH}_{3}\right), 2.28\left(\mathrm{~s}, 3 \mathrm{H}, \mathrm{CH}_{3}\right)$, $2.40-3.56\left(\mathrm{~m}, 6 \mathrm{H}, 3 \mathrm{CH}_{2}\right), 7.20-8.10(\mathrm{~m}, 5 \mathrm{H}, \mathrm{Ar}-\mathrm{H}), 10.95(\mathrm{~s}, 1 \mathrm{H}, \mathrm{NH}), 11.20(\mathrm{~s}$, 1H, OH). Anal. Cald. for $\mathrm{C}_{22} \mathrm{H}_{20} \mathrm{~N}_{6} \mathrm{O}_{3} \mathrm{~S}$ (448.42): C, $58.92 ; \mathrm{H}, 4.95$;N, 18.73; S, 7.13\%. Found: C, 58.89; H, 4.90; N, 18.70; S, 7.12.

\section{Cyclization of 12 a to give $14 a$}

To a stirred sodium ethoxide solution, prepared from sodium metal $(0.046 \mathrm{~g}$, $0.002 \mathrm{~mol})$ and absolute ethanol $(40 \mathrm{ml})$, compound $12 \mathrm{a}(0.85 \mathrm{~g}, 0.002 \mathrm{~mol})$ in ethanol/DMF was added. The mixture was refluxed while being stirred for $5 \mathrm{hr}$ and left to cool. The solid that precipitated was filtered off, washed with water, air dried and finally crystallized from $(\mathrm{EtOH})$ to give brown powder product that proved identical in all respects to 14 a (m.p.IR, yield 65\%) obtained above from 11 and $2 \mathrm{a}$.

2-(4-Acetyl-3-methel-6-phenylpyrazolo[3,4-c]pyrazol-1(6H)-yl)-3,5,6,7-tetrahydro$4 H$ - cyclopeta [4,5] thieno [2,3-d] pyrimidin - 4 -one (14a)

Brown crystals, Yield $(2.66 \mathrm{~g}, 62 \%), 201^{\circ} \mathrm{C}(\mathrm{EtOH})$. IR $(\mathrm{KBr}) \mathrm{v} \mathrm{cm}^{-1}: 3432$,

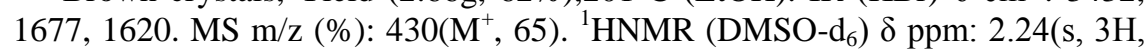


$\left.\mathrm{CH}_{3}\right), 2.32\left(\mathrm{~s}, 3 \mathrm{H}, \mathrm{CH}_{3}\right), 2.4-3.4\left(\mathrm{~m}, 6 \mathrm{H}, 3 \mathrm{CH}_{2}\right), 7.26-8.14(\mathrm{~m}, 5 \mathrm{H}, \mathrm{Ar}-\mathrm{H}), 10.88$ (s, $1 \mathrm{H}, \mathrm{NH})$. Anal. Calcd. For $\mathrm{C}_{22} \mathrm{H}_{18} \mathrm{~N}_{6} \mathrm{O}_{2} \mathrm{~S}$ (430.49): C, 61.38; H, 4.21; N, 19.52; S, $7.45 \%$. Found: C, 61.22; H, 4.19; N, 19.52; S, $7.46 \%$.

2-(4-Ethyl-3-methyl-6-phenylpyrazolo[3,4-c]pyrazol-1(6H)-yl)-3,5,6,7-tetrahydro $4 H$ - cyclopeta [4,5] thieno [2,3-d] pyrimidin -4-one-4-Carboxylate (14b)

Yellow crystals, Yield $(2.75 \mathrm{~g}, 60 \%)$, m.p $256{ }^{\circ} \mathrm{C}(\mathrm{EtOH}) . \mathrm{IR}(\mathrm{KBr}) v \mathrm{~cm}^{-1}$ : 310, 1738, 1665. MS m/z (\%): 460( $\left.\mathrm{M}^{+}, 52\right)$. ${ }^{1} \mathrm{HNMR}\left(\mathrm{DMSO}-\mathrm{d}_{6}\right) \delta \mathrm{ppm}: 1.45(\mathrm{t}$, $\left.3 \mathrm{H}, \mathrm{CH}_{3}\right), 2.25\left(\mathrm{~s}, 3 \mathrm{H}, \mathrm{CH}_{3}\right), 2.40-3.41\left(\mathrm{~m}, 6 \mathrm{H}, 3 \mathrm{CH}_{2}\right), 4.29\left(\mathrm{q}, 2 \mathrm{H}, \mathrm{CH}_{2}\right), 7.25-$ 8.11(m, 5H, Ar-H), 10.96(s, $1 \mathrm{H}, \mathrm{NH})$. Anal.Calcd. for $\mathrm{C}_{23} \mathrm{H}_{20} \mathrm{~N}_{6} \mathrm{O}_{3} \mathrm{~S}(460.43)$ : C, 59.48; H, 4.35; N, 18.10; S, 6.89\%. Found: C, 59.44; H, 4.38; N, 18.21; S, 6.91 $\%$.

2-(N-Phenyl-3-methyl-6-phenylpyrazolo[3,4-c]pyrazol-(6H)-yl)-3,5,6,7-tetrahydro4H-cyclopenta[4,5]thieno]2,3-d]pyrimidin-4-one-4-carboxamide (14c)

Brown crystals, Yield $(3.45 \mathrm{~g}, 62 \%)$ m.p. $186{ }^{\circ} \mathrm{C}(\mathrm{MeOH}) \mathrm{IR}(\mathrm{KBr}) \mathrm{v} \mathrm{cm}^{-1}$ : 3396-3128, 1695, 1625. MS m/z (\%): 507( $\left.\mathrm{M}^{+}, 46\right) .{ }^{1} \mathrm{HNMR}\left(\mathrm{CDCl}_{3}\right) \delta \mathrm{ppm}:$ 2.29(s, 3H, $\left.\mathrm{CH}_{3}\right), 2.31-3.00\left(\mathrm{~m}, 6 \mathrm{H}, 3 \mathrm{CH}_{2}\right), 7.10-8.12(\mathrm{~m}, 10 \mathrm{H}, \mathrm{Ar}-\mathrm{H}), 8.53(\mathrm{~s}, 1 \mathrm{H}$, $\mathrm{NH}), 10.96(\mathrm{~s}, 1 \mathrm{H}, \mathrm{NH})$. Anal. Calcd. for $\mathrm{C}_{27} \mathrm{H}_{21} \mathrm{~N}_{7} \mathrm{O}_{2} \mathrm{~S}$ (507.58): C, 63.89; H, 4.17; N, 19.32; S, 6.32\%. Found: C, 63.88; H, 4.20; N, 19.23; S, 6.31\%.

2- (3- Methyl-4-phenyl-6-phenylpyrazolo[ 3, 4-c ]pyrazol-1(6H)-yl)3,5,6,7-tetrahydro-4H-cyclopenta[4,5]thieno[2,3-d] pyrimidin - 4 - one (14d)

Yellow crystals, Yield $(2.92 \mathrm{~g}, 63 \%)$, m.p. $162{ }^{\circ} \mathrm{C}(\mathrm{EtoH})$. IR $(\mathrm{KBr}) \mathrm{v} \mathrm{cm}^{-1}$ : 3420, 1695. MS m/z (\%): 464( $\left.\mathrm{M}^{+}, 71\right) .{ }^{1} \mathrm{HNMR}\left(\mathrm{CDCl}_{3}\right) \delta \mathrm{ppm}: 2.23(\mathrm{~s}, 3 \mathrm{H}$, $\left.\mathrm{CH}_{3}\right), 2.41-3.50\left(\mathrm{~m}, 6 \mathrm{H}, 3 \mathrm{CH}_{2}\right), 7.11-8.00(\mathrm{~m}, 10 \mathrm{H}, \mathrm{Ar}-\mathrm{H}), 10.98(\mathrm{~s}, 1 \mathrm{H}, \mathrm{NH})$. Anal. Calcd. for $\mathrm{C}_{26} \mathrm{H}_{20} \mathrm{~N}_{6} \mathrm{OS}$ (464.26): C, 67.22; H, 4.34; N, 18.9; S, 6.90\%. Found: C, 67.23; H, 4.35; N, 18.9; S, 6.92\%.

2-(4-Benzoyl-3-methyl-6-phenyl pyrazolo[3,4-c]pyrazol-1(6H)-yl-3,5,6,7,4Hcyclopenta [4,5] thieno[2,3-d]Pyrimidin-4-one (14e)

Brown crystal, Yield $(2.85 \mathrm{~g}, 58 \%)$, m.p. $\left.186^{\circ} \mathrm{C} \mathrm{MeOH}\right)$. IR $(\mathrm{KBr}) \mathrm{v} \mathrm{cm}^{-1}$ : 3400, 1688, 1650. MS m/z (\%) 492( $\left.\mathrm{M}^{+}, 64\right) .{ }^{1} \mathrm{HNMR}\left(\mathrm{CDCl}_{3}\right) \delta \mathrm{ppm}: 2.22(\mathrm{~s}$, $\left.3 \mathrm{H}, \mathrm{CH}_{3}\right), 2.42-3.53\left(\mathrm{~m}, 6 \mathrm{H}, 3 \mathrm{CH}_{2}\right), 7.00-8.12(\mathrm{~m}, 10 \mathrm{H}, \mathrm{Ar}-\mathrm{H}), 10.98(\mathrm{~s}, 1 \mathrm{H}, \mathrm{NH})$. Anal. Calcd. for $\mathrm{C}_{27} \mathrm{H}_{20} \mathrm{~N}_{6} \mathrm{O}_{2} \mathrm{~S}$ (492.56): C, 65.84; H, 4.09; N, 17.06; S, 6.51\%. Found: C, 65.81; H, 4.12; N, 17.11; S, 6.53\%.

\section{References}

1. Shishoo, C. J., Devani, M.B., Bhadti, V.S., Jain, K.S., Rathod, I.S., Goyal, R.K., Gandhi, T.P., Patel, R.B. and Naik, S.R., Synthesis and pharmacological study of antihyperlipemic activity of 2-substituted thieno [2,3- $d$ ] pyrimidin - $4(3 H)$ - ones. Arzneim-Forsch, 40, 567-572 (1990).

2. Pathak, U.S., Singh, S. and Padh. J., Synthesis and biological activities of some 2$(N, N$-disubstituted) amino - 3 - phenylthieno [2,3-d] pyrimidin - 4 - $(3 H)$ ones. Indian J. Chem. Section B 30, 618-619 (1991).

Egypt. J. Chem. 55, No. 2 (2012) 
3. El-Sherbeny, M.A., El-Ashmawy, M. B., El-Subbagh, H. I., El-Emam, A. A. and Badria, F.A., Synthesis, antimicrobial and antiviral evaluation of certain thienopyrimidin derivatives. Euro. J. Med. Chem. 30, 445-449 (1995).

4. Darias, V., Abdallah, S.S., Tello, M.L., Delgado, L. D. and Vega, S., NSAI activity study of 4 - phenyl - 2 - thioxo - benzo [4,5] thieno [2,3-d] pyrimidin derivatives. Arch. Pharm. (Weinheim, Ger) 327, 779-783 (1994).

5.Russell, R. K., Press, J. B., Rampulla, R. A., McNally, J. J., Falotico, R., Keiser, J.A., Bright, D.A. and Tobia, A., Thiophene systems. 9. Thienopyrimidinedione derivatives as potential antihypertensive agents. J. Med. Chem. 31, 1786-1793 (1988).

6. Newberry, R. A. and Bushell, B. J., Thiazolo derivatives. U.S. 3, 979, 40: 07 D Sep (1976) Brit. Appl. 74/8, 870, Chem. Abs. 86, 29795z. Feb. (1977).

7. Sauter, F., Deinhammer, W. and Stanetty, P., Cyclisierungsreaktionen zu thiazolo $[3,2-a]$ thieno $[2,3-d]$ pyrimidinen. Monatch. Chem. 105, 1258-1265 (1974).

8. Shishoo, C.J. and Jain, K.S., Synthesis of some novel azido/ tetrazolothienopyrimidines and their reduction to 2, 4-siaminothieno [2,3-d] pyrimidines. J. Heterocyclic Chem. 29, 883-893 (1992).

9. Mahran, A. M. and Hassan N. A., One step synthesis and antimicrobial activities of new substituted sihydro-1,3,4-thiadiazoles. Arch. Pharm. Res. 29 (1), 46-49 (2006).

10. Shawali, A.S., Mahran, A.M. and Nada. A.A., Synthesis and antimicrobial activity of new functionalized derivatives of $[1,2,4]$ triazolo[4,3-a]pyrimidin-5(1H)-one. $J$. Heteroatom Chemistry, 18 (4), 393-398 (2007).

11. Abu-Zied, Kh .M., Hussein, H. A. R. and Abu-Hashem, A. A., Facile synthesis of polynuclear hetercycles and acyclic c-Nucleosides via $\alpha$-substituted cinamonitrile (II). In press in: Phosphorus Sulfur and Silicon(2011).

12. Eweiss, N.F. and Osman, A., Synthesis of heterocyclic .Part II, New routes to acetylthiadiazolines and alkylazothiazoles (1). J. Het. Chem. 17, 1713-1717 (1980).

13. Lozinskü, M.O., Kukota, S. K. and Pelkis, P.S., Ethylarylazochloroacetates and their reactions with morpholine and hydrazine hydrate. Khim. Zh. 33, 1295-1296 (Russ) (1967).

14. Shawali, A.S. and Osman, A., Synthesis and reactions of phenyl carbonyl arylhydrazidic chlorides. Tetrahedron. 27, 2517-2528 (1971).

15. Wolkoff, P., New method of preparing hydrazonoyl halides. Can. J. Chem. 53, 13331335 (1975).

16. Bunnett, J.F., Mechanism and reactivity in aromatic nucleophilic substitution reactions". Quart. Rev. (London). 12, 1-16 (1958).

17. Katsuyuti Ishii, Minoru Hatanaka and Ikuo Ueda, Smiles rearrangement of 2-(1methyl - tettrazol - 5 - thio) acetamides and their sulfonyl derifatives. Chem. Pharm. Bull. 39, 3331(1991) 
18. Aly, A.S., Abu-Zied, Kh. M. and Gaffer, Alaa El-Din, M., Synthesis and reactions of some novel azolothienopyrimidines and thienopyrimido as triazines derivatives. Phosphorus Sulfur and Silicon, 182, 447-474 (2007).

19. Bauer, A.W., Kirby, W.M, Sherries, C. and Turck, M, Antibiotic susceptibility testing by a standardized single disk method. American Journal of Clinical Pathology, 45, 493-496 (1966).

20. Liebowitz, L. D., Ashbee, H. R., Evans, E. G. V., Chong, Y. Mallatova, Zaidi, N. and Gibbs, D., Global antifungal surveillance group. A two year global evaluation of the susceptibility of Candida species to fluconazole by disk diffusion. Diagn. Microbial. Infect. Dis. 4, 27-33 (2001).

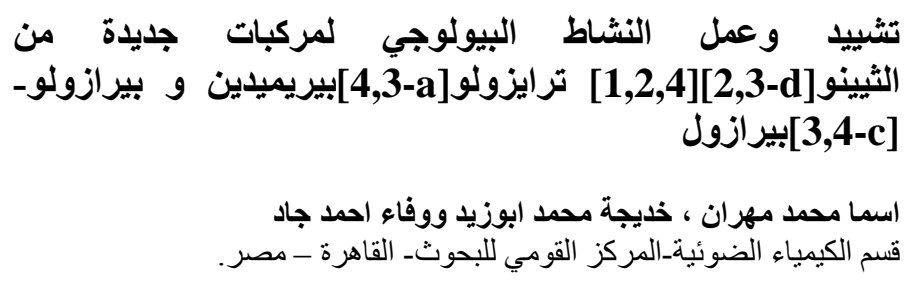

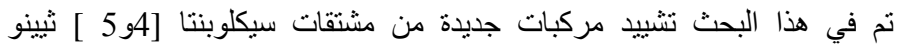

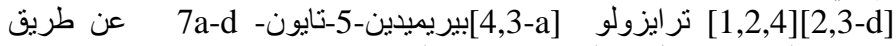

إضافة الهيدرازونويل هاليد

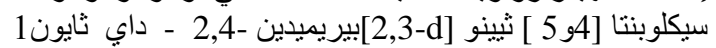

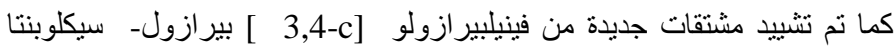

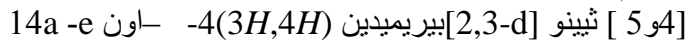

وتم التحقق من التركيب الكيميائي للمركبات المحضرة عن طريق أجر اء التحاليل الدقيقة للعناصر و أطياف الاشتعه تحت الحمر اء و الرنين المغناطيسي

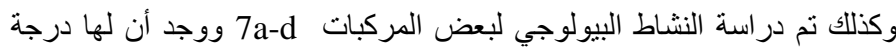

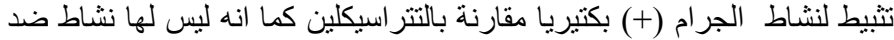
فطر اسبرجليسفلافيس أو كناد يدا البيكانس. 